\title{
Deep Learning-based Automatic Modulation Recognition Method in the Presence of Phase Offset
}

\author{
Jie Shi, Sheng Hong, Changxin Cai, Yu Wang, Hao Huang, and Guan Gui, Senior Member, IEEE
}

\begin{abstract}
Automatic modulation recognition (AMR) plays an important role in various communications systems. It has the ability of adaptive modulation and can adapt to various complex environments. Automatic modulation recognition is also widely used in orthogonal frequency division multiplexing (OFDM) systems. However, because the recognition accuracy of traditional methods to extract the features of OFDM signals is very limited. In order to solve these problems, many deep learning based AMR methods have been proposed to improve the recognition performance. However, most of these AMR methods neglect the harmful effect by carrier phase offset (PO) which often appears in real communications systems. Hence it is required to consider the PO effect for designing the OFDM system. Unlike conventional methods, we propose a convolutional neural network (CNN) based AMR method for considering PO in the OFDM system. The proposed method is used to eliminate the PO to achieve the high classification accuracy. Experiment results are provided to confirm the proposed method when comparing to conventional methods.
\end{abstract}

Index Terms-Deep learning, convolutional neural network, automatic modulation recognition, phase offset.

\section{INTRODUCTION}

Blind signal recognition is considered as one of important techniques in many military and civilian applications [1][8], such as adaptive modulator, spectrum sensing and noncooperative signal detection. Automatic modulation recognition (AMR) is a key step to realize the recognition technique and hence many AMR methods have been proposed in last decades. Generally speaking, these traditional AMR methods can be developed based on two types, i.e., likelihood function and feature extraction [9]. For one thing, the likelihood-based AMR method is formulated as a hypothesis testing problem [10] and hence it is necessary to design a correct likelihood function to find a likelihood for each modulation type within hypothesis pool. However, the likelihood-based AMR methods require accurate channel estimation of wireless channels. For another, the feature extraction based AMR method is formulated as a pattern recognition problem and it can be

This work was supported by the Project Funded by the National Science and Technology Major Project of the Ministry of Science and Technology of China under Grant TC190A3WZ-2, the Jiangsu Specially Appointed Professor under Grant RK002STP16001, the Innovation and Entrepreneurship of Jiangsu High-level Talent under Grant CZ0010617002, the Six Top Talents Program of Jiangsu under Grant XYDXX-010, the 1311 Talent Plan of Nanjing University of Posts and Telecommunications. (Corresponding author: Guan Gui)

J. Shi, S. Hong, Y. Wang, H. Huang and G. Gui are with the College of Telecommunications and Information Engineering, Nanjing University of Posts and Telecommunications, Nanjing 210003, China (e-mails: 546894976@qq.com, \{1018010405, 1018010407, 1017010502, guiguan\}@njupt.edu.cn)

C. Cai is with the School of Electronic and Information, Yangtze University, Jiangzhou 434023, China (email: caichangxinjpu@126.com). realized by pre-processing, feature extraction, and classifier design. However, these feature extraction-based AMR methods are considered as instantaneous realization scenarios, such as instantaneous features, wavelet transform-based features, high-order statistics-based features, cyclic spectrum analysisbased features, and so on. To classify the modulation types by extracted features [11], [12], they usually adopt various classifiers, such as high-order cumulants (HOC), support vector machine (SVM), decision tree (DT), $k$-nearest neighbor (KNN) and multilayer perception (MLP). According to aforementioned discussion, one may find that here traditional AMR methods require instantaneous information and realtime computational computing. Hence it is hard to apply these AMR methods in the next-generation heterogeneous communication systems [13]-[19].

Recently, deep learning (DL) has been attracted a lot of attentions due to its powerful ability to combine the offline training and online deployment [20]. In the perspective of data analysis, DL is expert in automatic feature extraction from big data, instead of the complex and difficult design of manmade features [21], [22]. Motivated by the advances, DL has been successfully applied in network traffic prediction [23]-[25], wireless communications [26]-[31], and internet of things [32]-[37].

Motivated by widespread applications, many DL-based AMR methods have been proposed to recognize different modulation signals in different systems. Among these deep learning methods, convolutional neural network (CNN) has been considered one of most effective methods due to excellence performance for extracting signal feature information in signal. CNN-based AMR methods have been proposed for various wireless communications systems due to their reliable performance [42]-[44]. For example, H. $\mathrm{Gu}$ et al. [38] proposed a blind channel identification aided generalized AMR method based on two independent convolutional neural networks (CNNs). S. Hong et al. [39] proposed a decision theory based AMR method for orthogonal frequency division multiplexing (OFDM) system. Note that OFDM technique can combat channel multipath fading by multi-carrier transmission and hence support highspeed parallel transmission by frequency division multiplexing [40]. In addition, because all of subcarriers are orthogonal, each sub-channel can overlap each other, which improves the frequency band utilization rate and is suitable for highspeed data transmission services. In the frequency domain, a given channel is divided into many orthogonal sub-channels. On each sub-channel, a sub-carrier is used for modulation, and each sub-carrier is transmitted in parallel, so as to 
effectively suppress inter-symbol interference (ISI) caused by time dispersion of wireless channels [41].

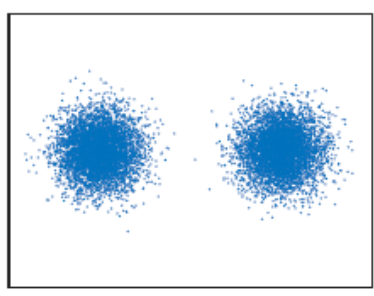

(a) phase offset $=0$

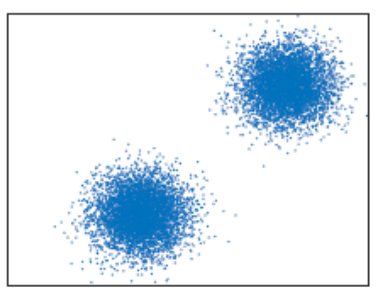

(b) phase offset $=\pi / 4$
Fig. 1. Example of constellation diagrams of BPSK signal in two phase offset cases.

However, these methods do not consider PO which is strongly deteriorate to signal detection at the receiver. A simple example of constellation diagrams of binary phase shift keying (BPSK) signal in two PO cases is shown in Fig. 1. PO is a very harmful factor in many multi-carrier communication systems [46] and radar signal systems [47]-[53].

In view of the excellent performance of $\mathrm{CNN}$ in the famous ImageNet large scale visual recognition challenge (ILSVRC) [54], this paper introduces CNN into AMR to complete the classification task. Among the two methods of AMR, the method of decision theory requires obtaining prior information and using probability theory and hypothesis testing theory to identify modulation types. This method is complex in calculation but simple in classification. Statistical pattern recognition can reflect the difference between the two, by extracting the characteristics of different modulation methods, and no prior knowledge is required. In addition, an appropriate classifier should be designed for modulation recognition according to the extracted features. At present, the latter method is the most important and effective one. In this paper, we propose CNN-based AMR method by considering the PO effect. At first, our proposed $\mathrm{CNN}$ is trained by inphase and quadrature (IQ) components of the received signal containing received signals at different SNRs by considering PO effects. We conduct the experiments to validate the proposed CNN-based AMR method. Several typical results show that our proposed can achieve comparable performance of the ideal CNN-AMR method without PO.

The rest of this paper is organized as follows. Second II introduces problem formulations including system model, signal model, dataset training and $\mathrm{CNN}$ architecture. Section III presents our proposed CNN-based AMR method. Section IV conducts experiments and evaluates our proposed method in different modulations. Finally, Section V concludes our work.

\section{Problem Formulation}

\section{A. System Model}

In this paper, a typical AMR-aided OFDM system is considered. The system model is shown in Fig. 2. In the whole system model, OFDM transmitter serves as the sending terminal. Firstly, the data stream through the modulator is converted in series and parallel, and the frequency domain signal is converted into a time-domain signal by means of inverse fast Fourier transformation (IFFT) for modulation. In addition, a cyclic prefix (CP) is added to reduce the intersymbol interference (ISI) between sub-channels. Finally, the parallel data stream is converted into serial data stream and the output is OFDM signal.

Then, the generated OFDM signal is sent to receiver, passing through channel and adding additive white Gaussian noise (AWGN). The signal needs to be pre-processed and signal-to-noise ratio (SNR) estimation is conducted. In this paper, a non-cooperative communication system is considered. That is to say, receiver cannot get any prior information about received signal, including modulation types. Hence, CNN-based AMR is introduced into the correct recognition of modulation types of received signals. After recognized signal modulation type, then it can be demodulated. Next, we briefly introduce the signal model and corresponding dataset generation for neural network training.

\section{B. Signal Model and Dataset Generation}

Here, single input single output (SISO) system is considered and its received signal model is given as

$$
r_{n}=\alpha e^{j \varphi_{c}} s_{n}+w_{n},
$$

where $\varphi_{c}$ denotes the $\mathrm{PO}$ and it range is usually set as $\varphi_{c} \in[0, \pi] . w_{n}$ stands for additive white Gaussian noise (AWGN), i.e., $w_{n} \sim C N(0,1) .\left\{r_{n}\right\}_{n=0}^{N-1}$ refers to the discrete time complex signal sequence sampled by Nyquist criterion. $s_{n}$ denotes one symbol in modulation signal, and $\left\{s_{n}\right\}_{n=0}^{N-1}$ has unit energy for fair comparison of different modulation types. $\alpha$ represents channel attenuation factor, and it is constant, when considering a time invariant system. Besides, nonideal synchronization problem is considered in order to make simulation closer to actual communication. In this paper, $\mathrm{PO}$ is the main influence of non-ideal synchronization to be considered. PO mainly results of propagation delay and initial carrier phase and assuming that $\mathrm{PO}$ obeys uniform distribution, i.e., $\varphi_{c} \sim U(0, \theta], 0 \leq \theta \leq \pi$. It is noted that $\mathrm{PO}$ is constant at the same observation phase. IQ samples are applied to train the former CNN. Through OFDM modulation, the $i$-th sampling data we obtained can be represented by a complex vector as

$$
\boldsymbol{s}_{i}=\left[s_{0}, s_{1}, \cdots, s_{n}, \cdots, s_{N-1}\right]
$$

where $n$ represents the number of sample points. At the same time, $s_{n}$ represents the value of the Nyquist sampling point, which is a complex number. $s_{n}$ can be expressed as

$$
s_{i}=\operatorname{Re}_{n}+j \operatorname{Im}_{n},
$$

where $\operatorname{Re}_{n}$ represents the real part of the $n$-th sampling point and $\operatorname{Im}_{n}$ represents its imaginary part of the signal $s_{n}$. They are the in-phase and orthogonal components of the signal respectively. It also represents the I's and Q's in the IQ sample. It is worth noting that $\boldsymbol{R}_{I Q}$ is a real matrix with dimensionality $2 \times N$ and it is the input of neural network. 


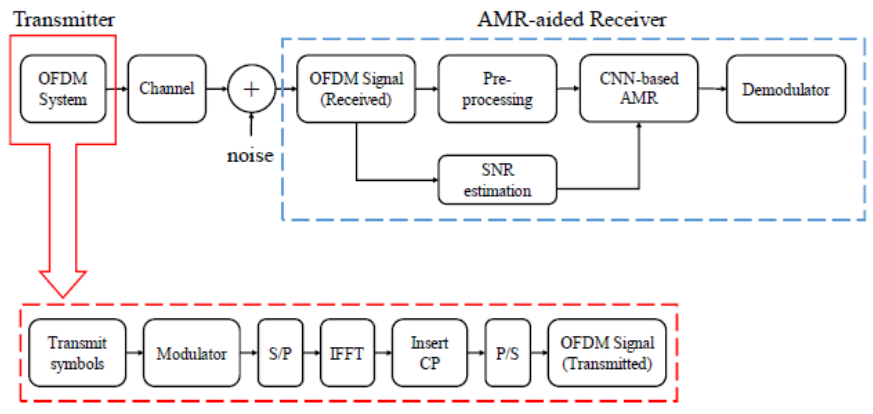

Fig. 2. Framework of the proposed CNN-based AMR system.

\section{The Proposed DL-BAsed AMR Method}

\section{A. CNN for AMR method}

In this section, a DL-based AMR method is proposed for uncooperative OFDM systems. The core of this method is CNN because of its excellent performance in feature extraction. It has been widely used in many fields, especially in the field of image processing.

A simple CNN model consists of an input layer, an output layer, a pooling layer, a fully connected layer, and an output layer. The convolutional layer is mainly used to extract the features of the input data and has been processed in the next step. The pooling layer can be used to improve accuracy by ignoring changes between the relative positions of the data. On the other hand, the pooling layer can reduce the feature dimension to avoid overfitting to a certain extent. The fully connected layer functions as a classifier in the entire CNN model. It uses the results of the previous layers to classify the input data to achieve the function of the CNN model. In this paper, modulation modes used by the received OFDM signal are classified through the fully connected layer, so as to effectively identify and facilitate subsequent demodulation work.

Every layer in CNN except the input layer needs a suitable activation function. More commonly used are rectified linear unit $(\mathrm{ReLu})$ activation function and parametric rectified Linear Unit (PReLu) activation function. As the name suggests, PReLu is ReLu with parameters. ReLU and PReLu activation function formulas are as follows:

$$
\begin{gathered}
\operatorname{Re} L u(x)= \begin{cases}x & x>0 \\
0 & x \leq 0,\end{cases} \\
\operatorname{PReLu}(x)=\left\{\begin{array}{ll}
x & x>0 \\
a x & x \leq 0
\end{array},\right.
\end{gathered}
$$

The sparse model implemented by PReLU can better mine related features and fit the training data. In addition, compared to other activation functions, PReLU has the following advantages: For linear functions, PReLU has a better effect, especially in deep neural networks. For non-linear functions, PReLU does not have a Vanishing Gradient Problem because the gradient of the non-negative interval is constant, so that the convergence speed of the model is maintained in a stable state.

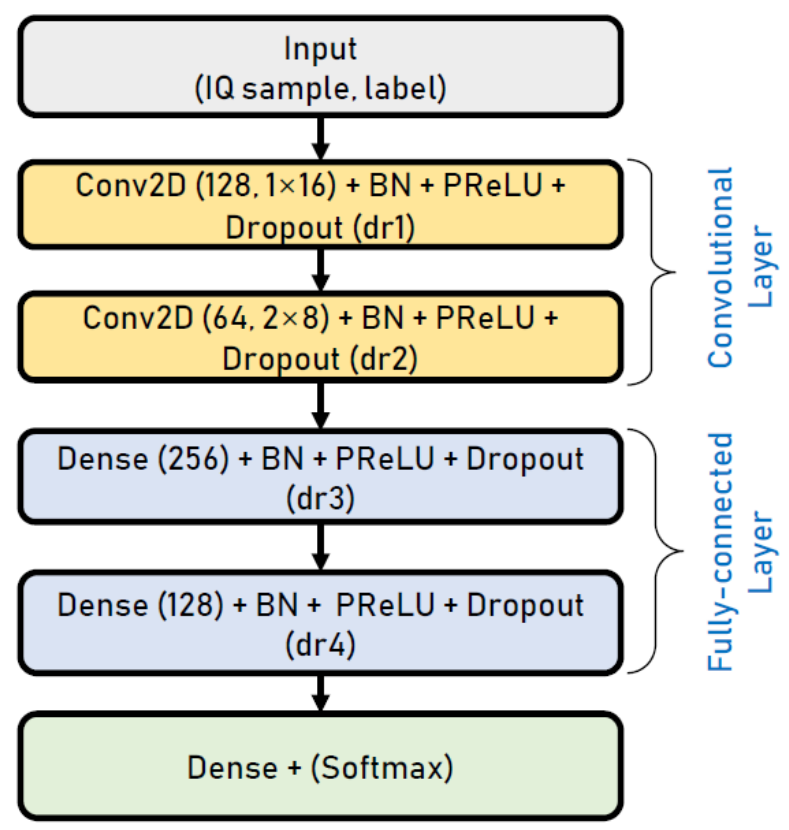

Fig. 3. CNN structure design in the proposed CNN-based AMR method.

Softmax regression model is a generalization of logistic regression model on multi-classification problems. It is applicable to multi-classification problems where the categories are mutually exclusive.

Suppose there is an array $E, e^{i}$ represents the $i$-th element in $E$, then the softmax value of the $e^{i}$ element is:

$$
S_{i}=\frac{e^{i}}{\sum_{j} e^{j}},
$$

The Softmax activation function maps the output of multiple neurons into the $(0,1)$ interval. It can be seen as the probability that the current output belongs to each classification, thereby completing the multi-classification task.

\section{B. The Proposed DL-based AMR Method}

As shown in Fig. 3, the proposed DL-based AMR method is mainly implemented by $\mathrm{CNN}$, which includes two convolutional layers and three fully connected layers. There are 128 convolution kernels in the first convolution layer, and the size of each convolution kernel is $1 \times 16$. The second convolution layer contains 64 convolution kernels, each of which is $2 \times 8$ in size. The number of neurons in the three fully connected layers is 256,128 , and $n$, where $\mathrm{n}$ represents the number of modulation modes. The PReLU function is used for all layers except the last layer, and the softmax function is used for the last layer to implement a multi-classification problem.

From Fig. 3, it can also been intuitively seen that each layer except the last layer has a batch normalization $(\mathrm{BN})$ layer and a dropout layer. The BN layer has been widely used in major networks, which has the effect of accelerating the speed of network convergence and improving training stability. It essentially solves the gradient problem in the process of backpropagation. That is, the BN layer forcibly pulls the input 
distribution of the relevant data back to a relatively standard normal distribution with a mean of 0 and a variance of 1 . The input value of the non-linear transformation function is made to fall into a region that is more sensitive to the input, thereby avoiding the problem of gradient disappearance.

When a complex feedforward neural network is trained on a small data set, it is easy to cause overfitting. To prevent overfitting, a certain neuron will stop working with a certain probability during forward propagation. The performance of neural networks can be improved by preventing the common response of feature detectors. This also makes the model more general, because the model does not rely too much on some local features.

\section{Algorithm Description}

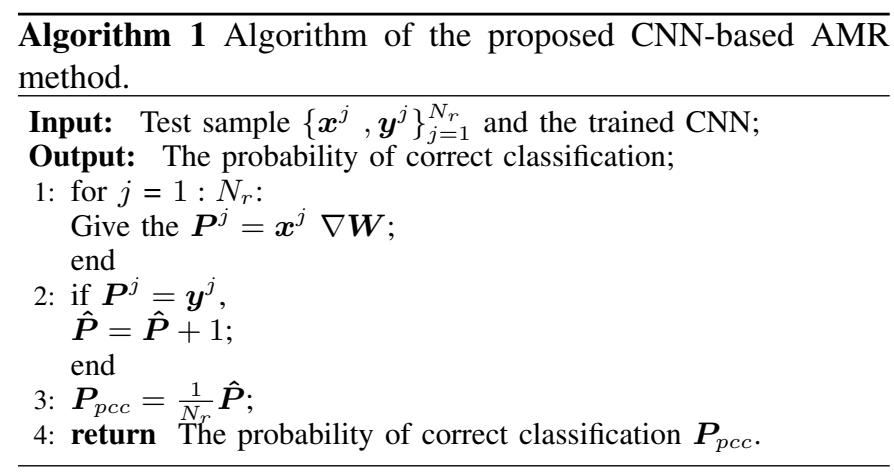

The algorithm description of the proposed CNN-based AMR method is shown in Algorithm 1. Known test samples $\left\{\boldsymbol{x}^{j}, \boldsymbol{y}^{j}\right\}_{j=1}^{N_{r}}$, where $\boldsymbol{x}^{j}$ is the data of the $j$-th sample, and $\boldsymbol{y}^{j}$ is the label value of the $j$-th sample, that is, the modulation type. Traverse $N_{r}$ samples and calculate the predicted label of the $j$-th sample as $\boldsymbol{P}^{j}$. Where $W$ is the weight of the nerual network, and $\nabla$ represents the calculation of the data from input to output. By comparing the values of the predicted label and the actual label, the number of samples with accurate prediction $\hat{\boldsymbol{P}}$ is calculated. Finally, the probability of correct classification of the proposed CNN-based AMR method $\boldsymbol{P}_{p c c}$ is obtained.

\section{Dataset Generation and Data Training}

For the AMR problem, we generate two data sets with different modulation modes. Dataset 1 consists of three modulation modes, which are BPSK, quadrature phase shift keying (QPSK), and eight phase shift keying (8PSK). Dataset 2 contains BPSK, QPSK, 8PSK, and sixteen quadrature amplitude modulation (16QAM). In our experiments, the OFDM system with 16 sub-carriers, 6 symbols of each subcarrier, CP of length 2 and 256-point FFT is considered. Taking BPSK modulation as an example, an initial signal is first generated. Then perform BPSK modulation, add subcarriers, and use inverse fast Fourier transform (IFFT) to convert the frequency domain signals into time domain signals. After that, the cyclic prefix and phase offset are added in turn. Finally, the received OFDM signal is obtained through Gaussian white noise.
TABLE I

EXPERIMENT PARAMETERS AND SETTING.

\begin{tabular}{|c|c|}
\hline Parameter & Value \\
\hline Style of the training & $\begin{array}{c}\text { Training IQ samples at each SNR }=i \mathrm{~dB}, \\
\text { where } i \in\{-10,-5, \cdots, 20\}\end{array}$ \\
\hline The number of training & 20,000 samples/type/SNR \\
\hline The number of testing & 20,000 samples/type/SNR \\
\hline Batch size & 500 \\
\hline Epochs & 500 \\
\hline Optimize & Stochastic gradient descent (SGD) \\
\hline Dropout (dr1-dr2-dr3-dr4) & $0.6-0.6-0.6-0.6$ \\
\hline
\end{tabular}

A large number of OFDM signals obtained through different modulation methods are sent to our proposed CNN-based AMR model for training, and finally the weight values of each neuron in the model are obtained. Then use the test data to verify our proposed model. By comparing the recognition accuracy, we can intuitively find that our proposed method has a high accuracy, which will be introduced in detail in the next section.

\section{EXPERIMENT RESULTS AND DISCUSSIONS}

In this section, we performe several sets of comparative experiments to verify the performance of DL-based AMR in non-cooperative OFDM systems. Traditional AMR methods are mostly composed of feature extraction and classifier. In this paper we consider the use of artificial features, which are mainly composed of the HOC feature and the instantaneous feature of the signal. In the classifier section, we use decision tree (DT) and random forest (RF) in traditional classification algorithms. The other two neural network based methods are CNN and Deep Neural Network(DNN). In addition, we also consider the difference in performance between the proposed method and the traditional method when the signal has a phase offset. Simulation parameters and setting are listed in Table I. The simulation requires powerful computing resources, so it is conducted on the platform with one Intel i7-8750H CPU and one NVIDIA GTX 1080Ti GPU. The implementation of neural networks relies on Keras 2.2.2 with Tensorflow 1.10 and Python 3.6.5 as the backend. Moreover, Matlab R2018a is applied to build our datasets.

In the simulation experiments, we prepare two datasets. Dataset 1: $\{$ BPSK, QPSK, 8PSK $\}$ and dataset 2: $\{$ BPSK, QPSK, 8PSK, 16QAM $\}$. The robustness of the proposed method is analyzed by performance comparison on different data sets. All data samples range from $-10 \mathrm{~dB}$ to $20 \mathrm{~dB}$ with the interval of $5 \mathrm{~dB}$. The total dataset consists of 20,000 samples at each signal-to-noise ratio, which are divided into training samples and test samples by $7: 3$. $70 \%$ of the data is used for training the model, and $30 \%$ of the data is used for testing the model to evaluate the recognition accuracy of the proposed method. In addition, Stochastic gradient descent (SGD) is chosen to detect the loss value to prevent overfitting.

Fig. 4 shows the probability of correct classification (PCC) of the proposed DL aided AMR method in dataset1. It can be seen intuitively that the performance of our proposed method is better than the traditional method based on feature extraction with or without phase offset. In the case of no phase offset, 


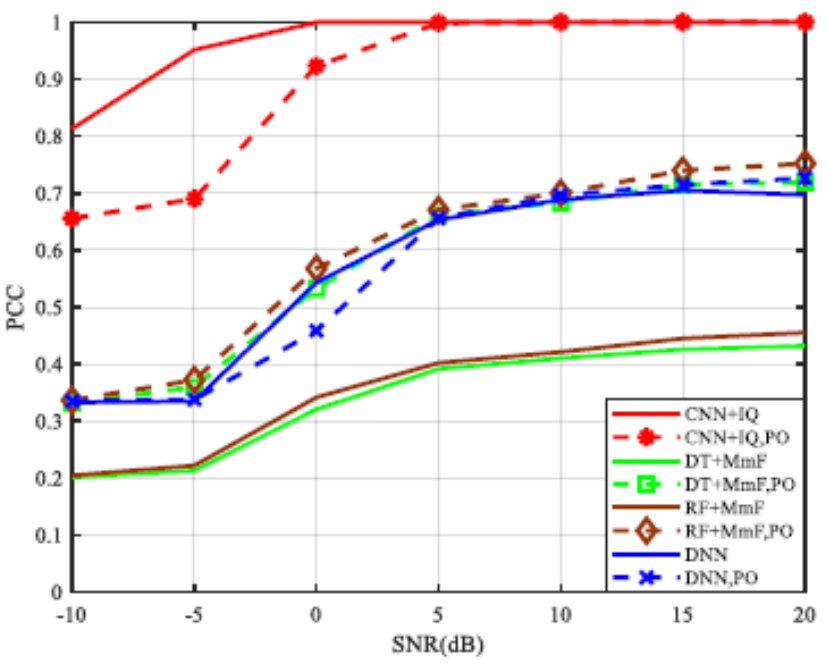

Fig. 4. Performance evaluations of these AMR methods in dataset 1: $\{$ BPSK, QPSK, 8PSK\}.

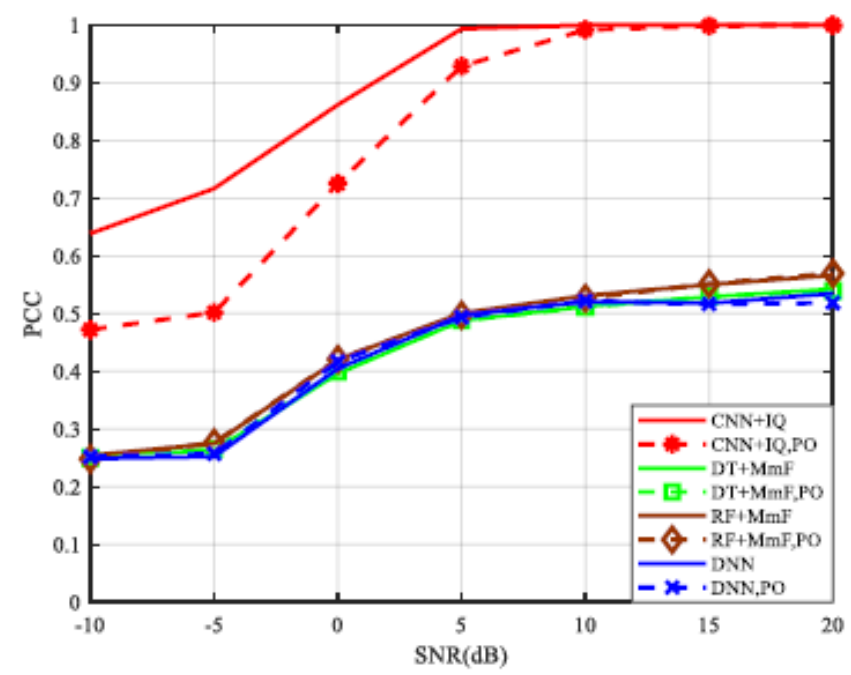

Fig. 5. Performance evaluations of these AMR methods in dataset 2: \{BPSK, QPSK, 8PSK, 16QAM\}.

when the SNR is $0 \mathrm{~dB}$, the recognition accuracy is as high as $100 \%$. Although the accuracy is slightly lower in the case of phase offset, almost no error recognition can be achieved when $\mathrm{SNR}=5 \mathrm{~dB}$. On the other hand, the PCC of the traditional method can only reach $70 \%$ under the high SNR. In Fig. 4, the curve trends of the traditional methods based on DT and RF are very similar, but the performance of the RF-based method is slightly better than that based on DT. This is because RF is composed of multiple DTs. When classifying and deciding, it will comprehensively consider the decisions of multiple DTs to give an overall decision. So the performance is better than DT to a certain extent. By comparing the CNN-based method and the DNN-based method, the superior performance of the proposed method can also be found intuitively. Because compared to DNN, CNN will first use the convolution layer to extract features of the signal. Then use the extracted features to recognize modulations. Taking into account the characteristics of the signal will help improving the accuracy of recognization.

In dataset 2, PCC of the proposed method is compared with the other three methods as shown in Fig. 5. Compared with curves in dataset 1 , when the SNR is less than $5 \mathrm{~dB}$, the recognition accuracy based on dataset 2 will be lower, and it is no exception when considering phase deviation. It can be seen that the introduction of 16QAM has caused some confusion to the original three modulation methods, so the recognition accuracy is lower. But when SNR gradually increases to $5 \mathrm{~dB}$, the PCC of proposed method can also reach $100 \%$. When SNR is $10 \mathrm{~dB}$, it can be recognized without error considering the phase offset. Therefore, combining the PCC of the proposed method and other traditional methods, we can find that the proposed DL aided method has high recognition accuracy and good robustness under different datasets.

\section{CONCLUSION}

In this paper, we have proposed a deep learning aided automatic modulation recognition method in the presence of phase offset in OFDM systems. This method is mainly implemented using CNN and training on IQ samples. It can accurately recognize four modulation modes: BPSK, QPSK, 8PSK, and 16QAM. Through comparative experiments, it is found that its recognition performance is far superior to the traditional feature extraction based methods. At the same time, the proposed method shows good robustness for different datasets. Although the introduction of phase offset will make the recognition accuracy slightly decrease at low SNRs, but with the gradual increase of SNRs, effective recognition can be achieved eventually. In future work, we plan to consider CNN aided AMR methods in multiple input multiple output systems (MIMO). More importantly, we try to further validate our proposed model using actual modulated signals instead of the ideal signals generated by Matlab.

\section{REFERENCES}

[1] N. Kato, B. Mao, F. Tang, Y. Kawamoto, and J. Liu,"Ten challenges in advancing machine learning technologies towards 6G," IEEE Wirel. Commun. Mag., in press, doi: 10.1109/MNET.001.1900476

[2] I. Bisio, C. Garibotto, F. Lavagetto, A. Sciarrone, and S. Zappatore, "Blind detection: Advanced techniques for WiFi-based drone surveillance," IEEE Trans. Veh. Technol., vol. 68, no. 1, pp. 938-946, 2020.

[3] G. Gui, H. Sari, and E. Biglieri, "A new definition of fairness for nonorthogonal multiple access," IEEE Commun. Lett., vol. 23, no. 7, pp. 1267-1271, 2019

[4] H. Gacanin, "Autonomous wireless systems with artificial intelligence: A knowledge management perspective," IEEE Veh. Technol. Mag., vol. 14, no. 1, pp. 51-59, 2019.

[5] F. Tang, Y. Kawamoto, N. Kato, and J. Liu, "Future intelligent and secure vehicular network towards 6G: Machine-learning approaches," Proc. IEEE, vol. 108, no. 2, pp. 292-307, 2020.

[6] M. Liu, J. Yang, and G. Gui, "DSF-NOMA: UAV-assisted emergency communication technology in a heterogeneous internet of things," IEEE Internet Things J., vol. 6, no. 3, pp. 5508-5519, 2019.

[7] I. Bisio, C. Garibotto, F. Lavagetto, and A. Sciarrone, "Outdoor places of interest recognition using WiFi fingerprints," IEEE Trans. Veh. Technol., vol. 68 , no. 5, pp. 5076-5086, 2019.

[8] X. Liu, Z. Li, N. Zhao, W. Meng, G. Gui, Y. Chen, and F. Adachi, "Transceiver design and multihop D2D for UAV IoT coverage in disasters," IEEE Internet Things J., vol. 6, no. 2, pp. 1803-1815, 2019.

[9] H. Wu, M. Saquib, and Z. Yun, "Novel automatic modulation classification using cumulant features for communications via multipath channels," IEEE Trans. Wirel. Commun., vol. 7, no. 8, pp. 3098-3105, 2008 . 
[10] F. Hameed, O. A. Dobre, D. C. Popescu, "On the likelihood-based approach to modulation classification," IEEE Trans. Wirel. Commun., vol. 8, no. 12, pp. 5884-5892, 2009.

[11] P. Li, F. Wang, and Z. Wang, "Algorithm for modulation recognition based on high-order cumulants and subspace decomposition," in International Conference on Signal Processing Proceedings (ICSP), Beijing, China, 2007, pp. 1-4.

[12] C. S. Park, J. H. Choi, S. P. Nah, W. Jang, and D. Y. Kim, "Automatic modulation recognition of digital signals using wavelet features and SVM," in International Conference on Advanced Communication Technology (ICACT), Gangwon-Do, Korea, 2008, pp. 387-390.

[13] Z. Zhou, X. Chen, and B. Gu, "Multi-scale dynamic allocation of licensed and unlicensed spectrum in software-defined HetNets," IEEE Netw., vol. 33, no. 4, pp. 9-15, Jul./Aug. 2019.

[14] Y.-J. Yu, T.-Y Hsieh and A.-C. Pang, "Millimeter-wave backhaul rraffic minimization for CoMP in 5G cellular networks," IEEE Trans. Veh. Technol., vol. 68, no. 4, pp. 4003-4015, 2019.

[15] Z. Zhou, J. Feng, Z. Chang, and X. Shen, "Energy-efficient edge computing service provisioning for vehicular networks: A consensus ADMM approach," IEEE Trans. Veh. Technol., vol. 68, no. 5, pp. 50875099, Mar. 2019.

[16] T. K. Rodrigues, K. Suto, and N. Kato,"Edge cloud server deployment with transmission power control through machine learning for 6G internet of things," IEEE Trans. Emerg. Topics Comput., in pres, doi: 10.1109/TETC.2019.2963091

[17] Z. Zhou, P. Liu, J. Feng, Y. Zhang, S. Mumtaz, and J. Rodriguez, "Computation resource allocation and task assignment optimization in vehicular fog computing: A contract-matching approach," IEEE Trans. Veh. Technol., vol. 68, no. 4, pp. 3113-3125, Apr. 2019.

[18] H. Gacanin, E. Perenda, and R. Atawia, "Self-deployment of nonstationary wireless systems by knowledge management with artificial intelligence," IEEE Trans. Cogn. Commun. Netw., vol. 5, no. 4, pp. 1004-1018, 2019

[19] Z. Zhou., H. Liao, X. Zhao, B. Ai, and M. Guizani, "Reliable task offloading for vehicular fog computing under information asymmetry and information uncertainty," IEEE Trans. Veh. Technol., vol. 68, no. 9, pp. $8322-8335,2019$

[20] G. E. Hinton, and R. R. Salakhutdinov, "Reducing the dimensionality of data with neural networks," Science, vol. 313, no. 5786, pp. 504-507, 2006.

[21] J. Sun, W. Shi, Z. Han, J. Yang, and G. Gui, "Behavioral modeling and linearization of wideband RF power amplifiers using BiLSTM networks for 5G wireless systems," IEEE Trans. Veh. Technol., vol. 68, no. 11, pp. 10348-10356, 2019.

[22] G. Gui, F. Liu, J. Sun, J. Yang, Z. Zhou, and D. Zhao, "Flight delay prediction based on aviation big data and machine learning," IEEE Trans. Veh. Technol., vol. 69, no. 1, pp. 1065-1069, 2020.

[23] F. Tang, B. Mao, Z. Md. Fadlullah, N. Kato, O. Akashi, and T.Inoue, and Kimihiro Mizutani, "On removing routing protocol from future wireless networks: A real-time deep learning approach for intelligent traffic control," IEEE Wirel. Commun. Mag., Vol. 25, no. 1, pp. 154$160,2018$.

[24] N. Kato, Z. Md. Fadlullah, B. Mao, F. Tang, O. Akashi, T. Inooue, and K. Mizutani, "The deep learning vision for heterogeneous network traffic control: Proposal, challenges, and future perspective," IEEE Wirel. Commun. Mag., vol. 24, no. 3, pp. 146-153, 2017.

[25] Z. Md. Fadlullah, F. Tang, B. Mao, N. Kato, O. Akashi, T. Inoue, K. Mizutani., "State-of-the-art deep learning: Evolving machine intelligence toward tomorrow's intelligent network traffic control systems," IEEE Commun. Surveys and Tuts., vol. 19, no. 4, pp. 2432-2455, 2017.

[26] H. Huang, J. Yang, H. Huang, Y. Song, and G. Gui, "Deep learning for super-resolution channel estimation and DOA estimation based massive MIMO system," IEEE Trans. Veh. Technol., vol. 67, no. 9, pp. 8549$8560,2018$.

[27] H. Huang, Y. Song, J. Yang, and G. Gui, "Deep-learning-based millimeter-wave massive MIMO for hybrid precoding," IEEE Trans. Veh. Technol., vol. 68, no. 3, pp. 3027-3032, 2019.

[28] Y. Wang, M. Liu, J. Yang, and G. Gui, "Data-driven deep learning for automatic modulation recognition in cognitive radios," IEEE Trans. Veh. Technol., vol. 68, no. 4, pp. 4074-4077, 2019.

[29] M. Liu, T. Song, J. Hu, J. Yang, and G. Gui, "Deep learning-inspired message passing algorithm for efficient resource allocation in cognitive radio networks," IEEE Trans. Veh. Technol., vol. 68, no. 1, pp. 641-653, 2019.

[30] G. Gui, H. Huang, Y. Song, and H. Sari, "Deep learning for an effective nonorthogonal multiple access scheme," IEEE Trans. Veh. Technol., vol. 67 , no. 9 , pp. 8440-8450, 2018.
[31] W. Wang, J. Yang, M. Liu, and G. Gui, "LightAMC: Lightweight automatic modulation classification using deep learning and compressive sensing," IEEE Trans. Veh. Technol., in press, doi: 10.1109/TVT.2020.2971001

[32] X. Sun, G. Gui, S. Member, and Y. Li, "ResInNet: A novel deep neural network with feature reuse for internet of things," IEEE Internet Things J., vol. 6, no. 1, pp. 679-691, 2019.

[33] M. Liu, T. Song, and G. Gui, "Deep cognitive perspective: Resource allocation for noma-based heterogeneous IoT with imperfect SIC," IEEE Internet Things J., vol. 6, no. 2, pp. 2885-2894, 2019.

[34] Z. Fadlullah et al., "State-of-the-art deep learning: Evolving machine intelligence toward Tomorrow's intelligent network traffic control systems," IEEE Commun. Surv. Tutorials, vol. 19, no. 4, pp. 2432-2455, 2017.

[35] J. Zhu, Y. Song, D. Jiang, H. Song, and S. Member, "A new deepQ-learning-based transmission scheduling mechanism for the cognitive internet of tThings," IEEE Internet Things J., vol. 5, no. 4, pp. 23752385,2018

[36] F. Tang, Z. M. Fadlullah, B. Mao, and N. Kato, "An intelligent traffic Load prediction based adaptive channel assignment algorithm in SDNIoT: A deep learning approach," IEEE Internet Things J., vol. 5, no. 6 , pp. 5141-5154, 2018.

[37] M. Mohammadi, G. S. Member, A. Al-fuqaha, S. Sorour, and M. Guizani, "Deep learning for IoT big data and streaming analytics: A survey," IEEE Commun. Surv. Tutorials, vol. 20, no. 4, pp. 2923-2960, 2018.

[38] H. Gu, Y. Wang, S. Hong, and G. Gui, "Blind channel identification aided generalized automatic modulation recognition based on deep learning," IEEE Access, vol. 7, no. 1, pp. 110722-110729, 2019.

[39] S. Hong, Y. Zhang, Y. Wang, H. Gu, G. Gui, and H. Sari, "Deep learningbased signal modulation identification in OFDM systems," IEEE Access, vol. 7, no. 1, pp. 114631-114638, 2019.

[40] B. A. Duel-hallen, "Fading channel prediction for mobile radio adaptive transmission systems," Proc. IEEE, vol. 95, no. 12, pp. 2299-2313, 2007.

[41] W. Yu and J. M. Cioffi, "FDMA capacity of Gaussian multiple-access channels with ISI," IEEE Trans. Commun., vol. 50, no. 1, pp. 102-111, 2002.

[42] F. Meng, P. Chen, L. Wu, and X. Wang, "Automatic modulation classification: A deep learning enabled approach," IEEE Trans. Veh. Technol., vol. 67, no. 11, pp. 10760-10772, 2018.

[43] Y. Tu and Y. Lin, "Deep neural network compression technique towards efficient digital signal modulation recognition in edge device," IEEE Access, vol. 7, no. 1, pp. 58113-58119, 2019.

[44] B. Tang, Y. Tu, Z. Zhang, and Y. Lin, "Digital signal modulation classification with data augmentation using generative adversarial nets in cognitive radio networks," IEEE Access, vol. 6, no. 1, pp. 15713-15722, 2018.

[45] D. Tjondronegoro and Y. P. Chen, "Using decision-tree to automatically construct learned-heuristics for events classification in sports video," in IEEE International Conference on Multimedia and Expo (ICME), Toronto, Canada, 2006, pp. 1465-1468.

[46] M. Marey, M. Guenach, and H. Steendam, "Code-aided channel tracking and frequency offset-phase noise elimination for multicarrier systems," IEEE Signal Proc. Lett., vol. 15, no. 11, pp. 657-660, 2008.

[47] F. Wen, J. Shi, and Z. Zhang, "Joint 2D-DOD, 2D-DOA and polarization angles estimation for bistatic EMVS-MIMO radar via PARAFAC analysis," IEEE Trans. Veh. Technol., in press,doi: 10.1109/TVT.2019.2957511

[48] F. Wen, J. Shi, and Z. Zhang, "Direction finding for bistatic MIMO radar with unknown spatially colored noise," Circuits Syst. Signal Process., in press, doi: 10.1007/s00034-019-01260-5

[49] F. Wen, C. Mao, and G. Zhang, "Direction finding in MIMO radar with large antenna arrays and nonorthogonal waveforms," Digit. Signal Process., vol. 94, pp. 75-83, Nov. 2019.

[50] X. Wang, L. Wan, M. Huang, C. Shen, and K. Zhang, "Polarization channel estimation for circular and non-circular signals in massive MIMO systems," IEEE J. Sel. Topics Signal Process., vol. 13, no. 5, pp. 1001-1016, 2019.

[51] X. Wang, D. Meng, M. Huang, and L. Wan, "Reweighted regularized sparse recovery for DOA estimation with unknown mutual coupling," IEEE Commun. Lett., vol. 23, no. 2, pp. 290-293, 2019.

[52] W. Fangqing, Z. Zhang, and G. Zhang, "Joint DOD and DOA estimation for bistatic MIMO radar: a covariance trilinear decomposition perspective," IEEE Access, vol. 7, no. 1, pp. 53273-53283, Apr. 2019. 
[53] W. Fangqing, Z. Zhang, K. Wang, G. Sheng, and G. Zhang, "Angle estimation and mutual coupling self-calibration for ULA-based bistatic MIMO radar," Signal Process., vol. 144, pp. 61-67, Mar. 2018.

[54] O. Russakovsky, et al., "ImageNet large scale visual recognition challenge," Int. J. Comput. Vis., vol. 115, no. 3, pp. 211-252, 2015.

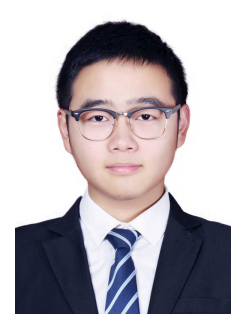

Jie Shi (S'18) received his B.S. Degree in Electronic Information Engineering from Nanjing University of Posts and Telecommunications (NJUPT) in 2017. He is currently working toward the master degree in NJUPT since 2018. His research interests include deep learning, optimization, and its application in wireless communications.

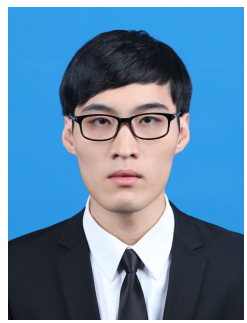

Sheng Hong (S'18) received his B.S. Degree in Electronic Information Engineering from Nanjing University of Posts and Telecommunications (NJUPT) in 2017. He is currently working toward the master degree in NJUPT since 2018. His research interests include deep learning, optimization, and its application in wireless communications

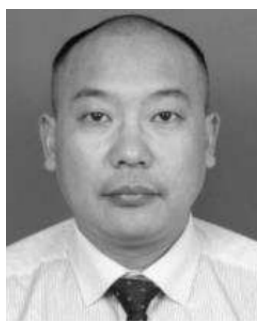

Changxin Cai was born in Hubei, China, in 1974. He received the B.S. degree in electronic instrument and measurement technology from Jianghan Petroleum University, Jingzhou, China, in 1998, the M.S. degree in geodetection and information technology from Jianghan Petroleum University in 2003, and the Ph.D. degree in petroleum and natural gas engineering from Yangtze University, China, in 2013. Since 2003, he has been with the Electronic and Information School, Yangtze University, where he is currently an Associate Professor, a Master Instructor, and the Associate Dean of the Electronic and Information School, mainly involved in signal and information processing, intelligent instrument design, measurement and control of oil and gas fields.

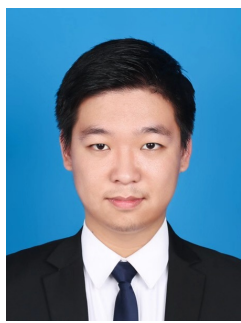

Yu Wang (S'18) received his B.S. degree in Communication Engineering from Nanjing University of Posts and Telecommunications (NJUPT), Nanjing, China in 2018. He is currently working toward the $\mathrm{Ph} . \mathrm{D}$. degree in NJUPT. Mr. Wang has published 8 international peer-reviewed journal/conference papers. He received several best paper awards, i.e., ICEICT 2019, CSPS 2019, CSPS 2018. His research interests include deep learning, optimization, and its application in wireless communications.

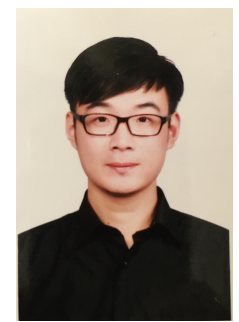

Hao Huang ( $\left.S^{\prime} 18\right)$ received the B.S. degree in the Photoelectric Information Science and Engineering from the Nanjing University of Posts and Telecommunications (NJUPT), Nanjing, China, in 2017. He is currently working toward the Ph.D degree in NJUPT. His research interests include deep learning, optimization, and its application in wireless communications.

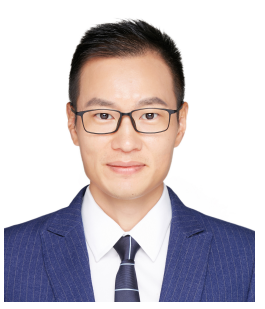

Guan Gui (M'11-SM'17) received the Ph.D degree from the University of Electronic Science and Technology of China, Chengdu, China, in 2012. From 2009 to 2014, he joined the Tohoku University as a research assistant as well as a postdoctoral research fellow, respectively. From 2014 to 2015, he was an Assistant Professor in the Akita Prefectural University. Since 2015, he has been a professor with Nanjing University of Posts and Telecommunications, Nanjing, China. His recent research interests include artificial intelligence, deep learning, non-orthogonal multiple access, wireless power transfer, and physical layer security.

$\mathrm{He}$ is an IEEE Senior Member. Dr. Gui has published more than 200 IEEE Journal/Conference papers and won several best paper awards, e.g., ICC 2017, ICC 2014 and VTC 2014-Spring. He received the Member and Global Activities Contributions Award in 2018, the Top Editor Award of IEEE TRANSACTIONS ON Vehicular TEChNOLOGY in 2019, the Exemplary Reviewer Award of IEEE COMMUNICATIONS LeTters in 2017. He was also selected as for the Jiangsu Specially-Appointed Professor in 2016 , the Jiangsu High-level Innovation and Entrepreneurial Talent in 2016, the Jiangsu Six Top Talent in 2018, the Nanjing Youth Award in 2018. He is serving or served on the editorial boards of several journals, including IEEE Transactions on Vehicular Technology, Physical Communication, Wireless Networks, IEEE ACCESs, Security and Communication Networks, IEICE Communications Express, and KSII Transactions on Internet and Information Systems, Journal on Communications. In addition, he served as TPC Chair of WiMob 2020, Track Chairs of VTC 2020 spring, ISNCC 2020 and ICCC 2020, Award Chair of PIMRC 2019, and TPC member of many IEEE international conferences, including GLOBECOM, ICC, WCNC, PIRMC, VTC, and SPAWC. 